\title{
Bread wheat grain yield and composition after a heatwave
}

\author{
Created by: Diana Tomás ${ }^{1}$, Jose Rodrigues ${ }^{2}$, Manuela Silva $^{3}$ \\ 1, Linking Landscape, Environment, Agriculture and Food (LEAF), Instituto \\ Superior de Agronomia, Universidade de Lisboa, Lisboa, Portugal; \\ dianarstomas@isa.ulisboa.pt \\ 2, Centro de Estudos Florestais (CEF), Instituto Superior de Agronomia, \\ Universidade de Lisboa, Portugal; jocarod@isa.ulisboa.pt \\ 3, Linking Landscape, Environment, Agriculture and Food (LEAF), Instituto \\ Superior de Agronomia, Universidade de Lisboa, Lisboa, Portugal; \\ manuelasilva@isa.ulisboa.pt
}

Version received: 26 April 2020

check for updates

Bread wheat is one of the most important cereals for food and feed and also one of the crops most affected by environmental changes. With the increasing frequency of extreme heat events become urgent to acquire a deeper understanding of their effects in wheat grain yield and nutritional parameters, such as protein content. The assessment of these parameters in distinct wheat varieties will enrich breeding programs contributing to assure food security. Comparative analysis of grains from control and treated plants disclosed intervarietal diversity in high temperature response regarding grain number, weight and macro components, such as protein. Moreover, both analyses showed that the intervarietal diversity observed in control conditions was significantly reduced in HT treated plants.

Wheat (Triticum aestivum L.) ensures $20 \%$ of the calories and $25 \%$ of proteins daily consumed worldwide. Although being produced under diverse environmental conditions, is one of the crops most affected by mean temperature increase during the growth season as each degree Celsius increase reduces wheat yield by $4.1 \%$ to $6.4 \%$ [1] . Several yield parameters are negatively affected by high temperatures as vegetative weight, grain number and weight (reviewed in ${ }^{[2]}$ ). Grain filling duration is also affected contributing to changes in starch and storage protein deposition ${ }^{[3]}$.

In this work, we aimed to evaluate the effect of a short-term high temperature (HT) treatment, imposed at the initial stages of grain filling, on grain yield and quality in distinct bread wheat varieties recommended to be produced in Portugal. The treatment imposed during grain filling mimeted a heatwave - a daily exposure of plants to a 4 hours period at $40 \circ \mathrm{C}$ during one week.

Comparative analysis between control and treated plants was performed and, for both yield and grain composition, intervarietal diversity in high temperature response was observed, with alterations on grain number and weight, and macro components, namely in protein content.

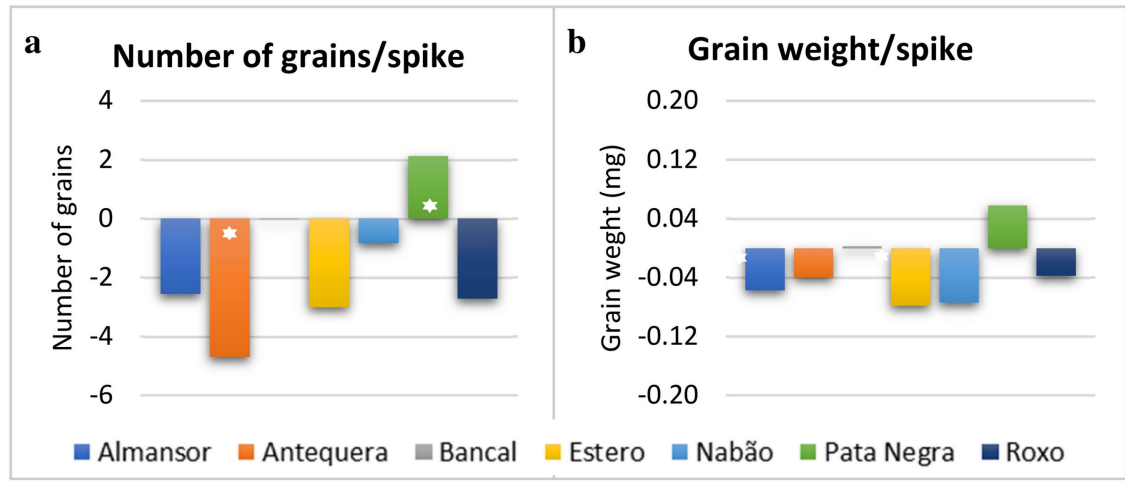


Figure 1) Grain yield. Changes in (a) number of grains/spike and (b) grains weight/spike of plants exposed to high temperature treatment during grain filling stage in comparison with plants kept in control conditions. $(*)$ indicates significant variation between control and treatment $(p<0.05)$.

Grain number and weight tended to decrease in the majority of the varieties analyzed. However, two varieties proved to be more resilient. Actually, Bancal and Pata Negra varieties presented promising yield results under HT treatment showing their potential for breeding strategies considering high temperature conditions (Figure $1 \mathrm{a}$ and $\mathrm{b}$ ).

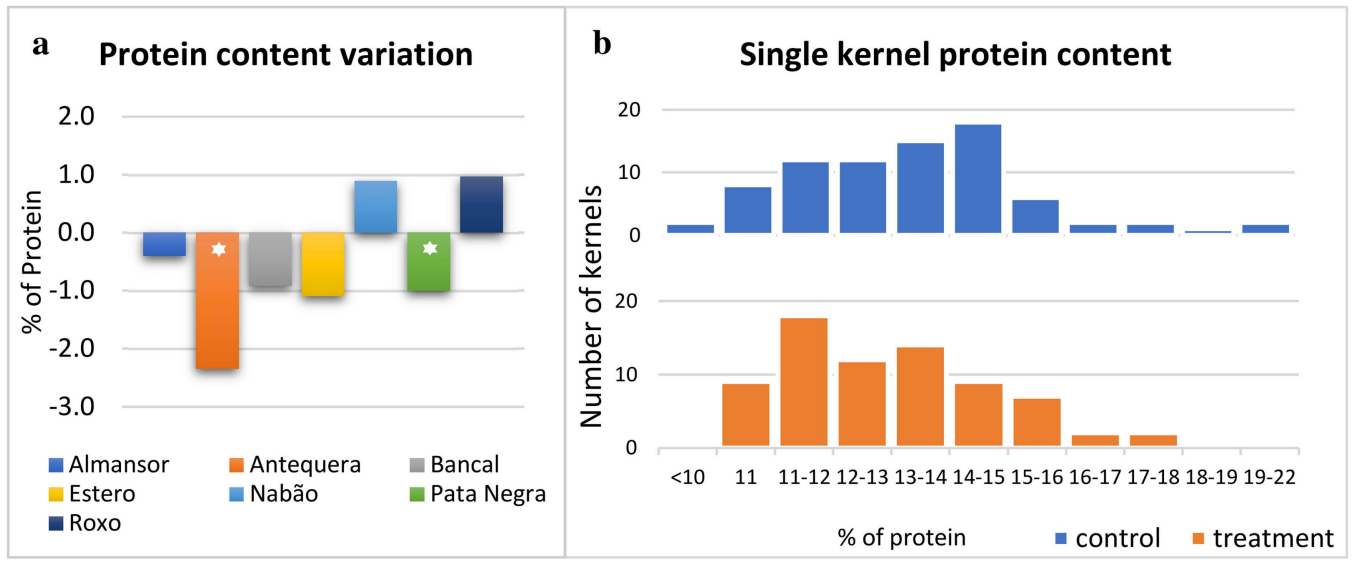

Figure 2) Protein content a) Changes in the average protein content induced by HT. b) Distribution of single grains protein content from untreated (blue) and high temperature treated (red) plants. $(*)$ indicates significant variation between control and treatment $(p<0.05)$.

Regarding protein content, varieties showed different responses to HT treatment (Figure 2a). In five varieties a decrease in protein content was observed, although it was significative only in two of them, Antequera and Pata Negra.

Also the range of single kernel protein content in grains from treated plants (between 10.1 and $17.6 \%$ ) is lower in comparison with the range observed in control ones (between 9.5 and $21.4 \%$ ) (Figure 2b), as well as the standard deviation ( $1.8 \%$ and $2.2 \%$, respectively). This evidence the reduction of intervarietal differences after HT treatments as previously observed in transcription levels and protein fractions proportions ${ }^{[\underline{4}]}$.

This work strongly contributes to heat response knowledge, namely regarding heatwaves, increasingly common and intense in Portugal during wheat grain filling. A deeper understanding of their effects in yield and nutritional parameters, such as protein content, urge in a time of climatic changes to enrich breeding programs of such a relevant crop worldwide as wheat.

\section{References}

1. Bing Liu; Senthold Asseng; Christoph Müller; Frank Ewert; Joshua Elliott; David B. Lobell; Pierre Martre; Alex C. Ruane; Daniel Wallach; James W. Jones; et al.Cynthia RosenzweigPramod AggarwalPhillip D. Aldermanjakarat AnothaiBruno BassoChristian BiernathDavide CammaranoA J ChallinorDelphine DeryngGiacomo De SanctisJordi DoltraElias FereresChristian FolberthMargarita Garcia-VilaSebastian GaylerGerrit HoogenboomLeslie K. HuntRoberto C. IzaurraldeMohamed JablounCurtis Dinneen JonesK.C. KersebaumB.A. KimballAnn-Kristin KoehlerSoora Naresh KumarClaas NendelGarry J. O'LearyJørgen Eivind OlesenMichael J. OttmanTaru PalosuoP.V.V. PrasadEckart PriesackThomas A. M. PughMatthew ReynoldsEhsan Eyshi RezaeiReimund P. RötterErwin SchmidMikhail Semenovlurii ShcherbakElke StehfestClaudio O. StöcklePierre StratonovitchThilo Strecklwan SupitFulu TaoPeter ThorburnKatharina WahaGerard W. WallEnli WangJeffrey W. Whitejoost WolfZhigan ZhaoYan Zhu Similar estimates of temperature impacts on global wheat yield by three independent methods. Nature Climate Change 2016, 6, 11301136, 10.1038/nclimate3115.

2. Nurunnaher Akter; M. Rafiqul Islam; Heat stress effects and management in wheat. A review. Agronomy for Sustainable Development 2017, 37, 17, 10.1007/s13593-017-0443-9.

3. Susan B. Altenbach; New insights into the effects of high temperature, drought and post-anthesis fertilizer on wheat 
grain development. Journal of Cereal Science 2012, 56, 39-50, 10.1016/j.jcs.2011.12.012.

4. Diana Tomás; Wanda Viegas; Manuela Silva; Effects of Post-Anthesis Heat Waves on the Grain Quality of Seven European Wheat Varieties. Agronomy 2020, 10, 268, 10.3390/agronomy10020268.

\section{Keywords}

bread wheat; heatwave; yield; grain composition; protein content

\section{Dol Information}

DOI: 10.32545/encyclopedia202005.0012.v1 | 셔 PDF [ 859kb, updated 24 June 2020 ]

(c) (i) (C) 2020 by the author(s). Distribute under a Creative Commans CC BY license 